\title{
A Hamiltonian Formulation for Long Internal Waves
}

\author{
Yuri Lvov* \\ Department of Mathematical Sciences \\ Rensselaer Polytechnic Institute
}

\author{
Esteban G. Tabak ${ }^{\dagger}$ \\ Courant Institute of Mathematical Sciences \\ New York University
}

October 22, 2018

\begin{abstract}
A novel canonical Hamiltonian formalism is developed for long internal waves in a rotating environment. This includes the effects of background vorticity and shear on the waves. By restricting consideration to flows in hydrostatic balance, superimposed on a horizontally uniform background of vertical shear and vorticity, a particularly simple Hamiltonian structure arises, which can be thought of as describing a nonlinearly coupled infinite collection of shallow water systems. The kinetic equation describing the time evolution of the spectral energy of internal waves is subsequently derived, and a stationary Kolmogorov solution is found in the high frequency limit. This is surprisingly close to the Garrett-Munk spectrum of oceanic internal waves.
\end{abstract}

\section{submitted to JFM}

\section{Introduction}

The term ocean waves typically evokes images of surface waves shaking ships during storms in the open ocean, or breaking rhythmically near the shore. Yet much of the ocean wave action takes place underneath the surface, and consists of modulations not of the air-water interface, but of invisible surfaces of constant density. These internal waves are ubiquitous in the ocean, contain a large amount of energy, and affect significantly the processes involved in water mixing and transport.

Our knowledge of the typical scales and energy content of oceanic internal waves advanced significantly through improved and more widespread observations in the last few decades. In particular, an empirically based formula that Munk and Garrett developed in the seventies (now called the Garrett Munk spectra of

\footnotetext{
*Corresponding author, e-mail is lvovy@rpi.edu

${ }^{\dagger} \mathrm{E}$-mail is tabak@cims.nyu.edu
} 
internal waves) synthesizes magnificently a seemingly universal distribution of energy among scales [1-3]. Description of modern observational work can be found in [4-8]. In particular, in [6] the deviation from the Garrett-Munk spectra are documented, and in [7] the dissipation rate of turbulent kinetic energy is measured. On the theoretical side, this distribution has been generally understood as due to the effects of nonlinear interaction among waves, amenable to a description based on kinetic equations analogous to the ones of statistical mechanics. Probably the first kinetic equation for internal waves was written in [9], though not within the frame of a Hamiltonian formalism.

A comprehensive review of a significant line of work developed in the seventies and eighties is provided in [10] and references therein. Some important references not cited there are [11-13]. More recent work includes, for example, [14] where a thorough perturbative Eulerian-Boussinesq approach in a nonrotating environment is developed. See [15-17] for a detailed discussion of the relation between the spectral tails of Lagrangian and Eulerian flow descriptions. One can also use ray theory to study internal wave scattering, as in [18], where the Doppler-spreading of short internal wave packets in the atmosphere and the ocean is studied or, as in [19], where the refraction of short oceanic internal waves by a spectrum of large amplitude inertia waves is considered.

Our work differs from the line reviewed in [10] in various ways. In [10] the wave dynamics is formulated in a fully Lagrangian framework, while our isopycnal formulation is Eulerian in the horizontal coordinates and Lagrangian in the vertical. To write down the equations of motion in the Lagrangian framework, the system's Lagrangian is expanded in powers of the assumed small displacement of the fluid parcels. This description is therefore approximate even at the level of the dynamic equations of motion. Such a description fails to adequately describe the advection of small scale waves by larger scale flows, as well as the interaction of waves with the vortical part of motion. This is acknowledged in [10], which proposes as a challenge the rederivation of the kinetic equation in an Eulerian framework. In the present article, we fulfill this program, and use therefore as a small parameter not the small displacement of fluid parcels, but the weakness of the nonlinear interaction among waves.

A fundamental question posed in the eighties is whether the GM spectra is close to the statistical 
steady state solution of the kinetic equation. The discussion in [10] indicates that GM is not inconsistent with the kinetic equation proposed, and may be close to being a steady state solution. Recently, the authors have put forward a study showing that power law spectra which, in the high frequency limit, are very close to GM, constitute exact analytical steady state solutions of a kinetic equation for hydrostatic flows described in isopycnal coordinates [20]. The present article extends those early results, by starting to build a theory that includes frequencies comparable to the rate of rotation of the Earth, and that accounts for the existence of large-scale horizontal eddies and vertical shear in the ocean, over which the internal waves are superimposed.

The main contribution of this paper is the development of a novel Hamiltonian formalism for the description of internal waves. Our approach is general enough to include the effects of the Earth's rotation, of large-scale eddies and of vertical shear on the waves, yet exclusive enough in its assumptions to yield a relatively simple, manageable model. The main assumption is that the waves are long enough to be in hydrostatic balance, yet they live in horizontal scales shorter than those characterizing the underlying eddies. This allows us to consider as unperturbed flow an arbitrary layered distribution of potential vorticity and vertical shear -that is, potential vorticity and horizontal velocity profiles which adopt independent, horizontally uniform values at each depth. Such hydrostatically balanced, horizontally uniform, vertically varying profiles are quite representative of long waves in the real ocean; they arise due to the highly anisotropic nature of the ocean's eddie diffusivity, which tends to homogenize the flow along isopycnal surfaces.

Hamiltonian structures for stratified incompressible flows have been a subject of active research over the last few decades. Though a complete review of the subject is outside the scope of this paper, we list here some of the most important results. The first paper to derive a Hamiltonian structure for stratified internal waves is probably [12], where a representation is proposed based on Clebsh-like variables. The resulting Hamiltonian is an explicit infinite power series of canonically conjugated variables. In [21], a Hamiltonian formalism for internal waves in isopycnal coordinates is developed. No hydrostatic approximation is invoked, and thus the resulting Hamiltonian is expressed as an explicit power-series in the 
powers of the assumed small nonlinearity. Potential problems in using Clebsh variables for stratified flows have been addressed in $[22,23]$. Problems of describing the Hamiltonian structures to describe interaction of wave and vortex mode is addressed in [24]. In addition to the references above, a noncanonical Hamiltonian structure based on a Lie-Poisson framework has been developed in [25]. More recently, two broad reviews on Hamiltonian structures for fluids have been published [26, 27].

We choose to describe the flow in isopycnal coordinates, replacing the depth $z$ by the density $\rho$ as the independent vertical coordinate. The advantage of such semi-Lagrangian description is manifold. First, it eliminates the need to handle the vertical velocity explicitly, which renders the equations much more tractable. At a deeper level, it greatly simplifies the description of the interaction between waves and vorticity, since potential vorticity is preserved along particle trajectories, and these remain on isopycnals in the absence of mixing. In particular, if the potential vorticity is uniform throughout an isopycnal surface, it remains so forever. This is the situations we choose to describe: a profile of vorticity which varies across surfaces of constant density, but is homogeneous along them. Such "pancake-like" profiles are quite representative of the intermediate scales in the ocean, smaller than the dominant eddies, but still containing a significant wealth of internal waves. Modeling this scenario constitutes an intermediate step between considering irrotational frameworks, and studying the fully turbulent interaction between arbitrary profiles of vorticity and waves.

Finally, the equations in isopycnal coordinates are highly reminiscent of the equations for a shallow single layer of homogeneous fluid. We exploit this formal analogy to develop a Hamiltonian formalism for internal waves which extends naturally similar formalisms for shallow waters.

The plan of this paper is the following. In section (2), we develop a hierarchy of Hamiltonian descriptions for long waves. Our goal here is to develop a general Hamiltonian formalism for nonlinear internal waves in a rotating environment, superimposed on a background of layered potential vorticity and horizontally uniform shear. However, we choose to do so in stages, starting in the simple context of linear, irrotational shallow water equations, and adding progressively nonlinearity, stratification, the Coriolis effect, and nontrivial potential vorticity. This not only clarifies the logic and essential simplicity 
of the structure of the final Hamiltonian, but also yields along the way a series of Hamiltonian structures for problems of intermediate simplicity. In section (3), we derive kinetic equations for the time evolution of the energy spectrum of internal waves, based on the Hamiltonian description developed in the previous section. For this, we constrain ourselves to consider a neutral background with neither shear nor vorticity. However, we still include the Coriolis effect. Then, in section (4), we find approximate stationary solutions to the kinetic equations corresponding to a direct cascade of energy toward the finest scales, in the high-frequency limit, where the effects of the Earth's rotation are negligible. This was the situation considered in [20]. Finally, in section (5), we provide some concluding remarks, and discuss open problems for further research.

\section{Hamiltonian formalism for long internal waves}

In this section we develop a Hamiltonian formalism for long internal waves in a rotating environment. We choose to do so progressing through a hierarchy of models, which starts with linear, irrotational shallow water waves in a non-rotating environment, and ends up with fully nonlinear internal waves in a stratified and rotating environment, superimposed on an arbitrary layered distribution of potential vorticity.

\subsection{Linear, Non-Rotating Shallow Waters}

In non-dimensional form, the shallow-water equations take the form

$$
\begin{aligned}
h_{t}+\nabla \cdot(h \vec{u}) & =0, \\
\vec{u}_{t}+(\vec{u} \cdot \nabla) \vec{u}+\nabla h & =0 .
\end{aligned}
$$

Here $h$ represents the height of the free-surface, and $\vec{u}$ the horizontal velocity field. The height $h$ has been normalized by its mean value $H$, the velocity field $u$ by the characteristic speed $c=\sqrt{g h}$ (here $g$ is the gravity constant), the horizontal coordinates by a typical wavelength $L$, and time by $L / c$. Writing

$$
h=1+\eta,
$$


and assuming that $\eta$ and $|u|$ are much smaller than one, one obtains to leading order the linearized equations

$$
\begin{aligned}
\eta_{t}+\nabla \cdot \vec{u} & =0 \\
\vec{u}_{t}+\nabla \eta & =0 .
\end{aligned}
$$

At this linear level, the dynamics of waves and vorticity decouple, with the former satisfying the wave equation, and the latter remaining constant in time. In particular, if the flow is initially irrotational (i.e., $\nabla \times \vec{u}=0$ ), it will remain so forever. Hence we may restrict our attention here to irrotational flows. These may be described by a scalar potential $\phi$, such that

$$
\vec{u}=\nabla \phi .
$$

For such flows, the system in $(3,4)$ reduces to

$$
\begin{aligned}
\eta_{t}+\Delta \phi & =0, \\
\phi_{t}+\eta & =0 .
\end{aligned}
$$

This system is Hamiltonian, with

$$
\mathcal{H}=\frac{1}{2} \int\left(\eta^{2}+|\nabla \phi|^{2}\right) d x .
$$

The Hamiltonian form of the equations is

$$
\begin{aligned}
\eta_{t} & =\frac{\delta \mathcal{H}}{\delta \phi} \\
\phi_{t} & =-\frac{\delta \mathcal{H}}{\delta \eta} .
\end{aligned}
$$

Notice that the Hamiltonian in (5) is the sum of the potential and kinetic energy of the system. The former is actually given by $\frac{1}{2}(1+\eta)^{2}$, but the difference can be absorbed by a gauge transformation of the potential $\phi$. Our goal is to preserve the essential simplicity of this formulation when we add nonlinearity, ambient rotation and stratification. 


\subsection{Nonlinear, Non-Rotating Shallow Waters}

For the fully nonlinear shallow-water equations in $(1,2)$, waves and vorticity no longer decouple (in fact, the nonlinear interaction of waves and vorticity is among the main theoretical obstacles to a full description of turbulence.) However, it is still true that a flow which starts irrotational stays so forever. Hence we may restrict ourselves to considering this scenario, introduce again the scalar potential $\phi$, and rewrite $(1,2)$ in the form

$$
\begin{aligned}
& h_{t}+\nabla \cdot(h \nabla \phi)=0, \\
& \phi_{t}+\frac{1}{2}|\phi|^{2}+h=0 .
\end{aligned}
$$

This system is also Hamiltonian, with

$$
\mathcal{H}=\frac{1}{2} \int\left(h^{2}+h|\nabla \phi|^{2}\right) d x,
$$

and canonical equations

$$
\begin{aligned}
h_{t} & =\frac{\delta \mathcal{H}}{\delta \phi}, \\
\phi_{t} & =-\frac{\delta \mathcal{H}}{\delta h} .
\end{aligned}
$$

In this case, the Hamiltonian is the sum of the potential and kinetic energy, without qualifications.

\subsection{Nonlinear, Non-Rotating, Internal Waves}

The non-dimensional equations of motion for long internal waves in an incompressible, stratified fluid with hydrostatic balance, are given by

$$
\begin{array}{rlrl}
\frac{d \vec{u}}{d t}+\frac{\nabla P}{\rho} & =0, & & P_{z}+\rho=0, \\
\frac{d \rho}{d t} & =0, & \nabla \cdot \vec{u}+w_{z}=0,
\end{array}
$$


where $\vec{u}$ and $w$ are the horizontal and vertical components of the velocity respectively, $P$ is the pressure, $\rho$ the density, $\nabla=\left(\partial_{x}, \partial_{y}\right)$ the horizontal gradient operator, and

$$
\frac{d}{d t}=\frac{\partial}{\partial t}+\vec{u} \cdot \nabla+w \frac{\partial}{\partial z}
$$

is the Lagrangian derivative following a particle.

Changing to isopycnal coordinates $(x, y, \rho, t)$, where the roles of the vertical coordinate $z$ and the density $\rho$ as independent and dependent variables are reversed, the equations become:

$$
\begin{aligned}
\frac{D \vec{u}}{D t}+\frac{\nabla M}{\rho} & =0, \\
M_{\rho} & =z, \\
z_{\rho, t}+\nabla \cdot\left(z_{\rho} \vec{u}\right) & =0 .
\end{aligned}
$$

Here $\vec{u}=(u, v)$ is the horizontal component of the velocity field, $\nabla=\left(\partial_{x}, \partial_{y}\right)$ is the gradient operator along isopycnals, $\frac{D}{D t}=\partial_{t}+\vec{u} \cdot \nabla$, and $M$ is the Montgomery potential [28],

$$
M=P+\rho z
$$

For flows which are irrotational along isopycnal surfaces, we introduce the velocity potential

$$
\vec{u}=\nabla \phi
$$

Such a substitution allows us to integrate (10) once and illuminate $z$, after which these equations reduce to the pair

$$
\begin{aligned}
\phi_{t}+\frac{1}{2}|\nabla \phi|^{2}+\frac{1}{\rho} \int^{\rho} \int^{\rho_{2}} \frac{\Pi}{\rho_{1}} d \rho_{1} d \rho_{2} & =0, \\
\Pi_{t}+\nabla \cdot(\Pi \nabla \phi) & =0,
\end{aligned}
$$

where we have introduced the variable

$$
\Pi=\rho M_{\rho \rho}=\rho z_{\rho} .
$$


This variable $\Pi$ has at least two physical interpretations. One is that of density in isopycnal coordinates, since

$$
\Pi d \rho=\rho d z .
$$

The other is that of a measure of the stratification, namely the relative distance between neighboring isopycnal surfaces, since this distance $d z$ is given by

$$
d z=\Pi \frac{d \rho}{\rho} .
$$

Notice the similarity between (11) and the equations (8) for nonlinear shallow water. Internal wave equations could be viewed as a system of infinitely many, coupled shallow water equations. This analogy allows us to identify a natural Hamiltonian structure for internal waves.

The variable $\Pi$ is also the canonical conjugate of $\phi$,

$$
\Pi_{t}=\frac{\delta \mathcal{H}}{\delta \phi}, \quad \phi_{t}=-\frac{\delta \mathcal{H}}{\delta \Pi},
$$

under the Hamiltonian flow given by

$$
\mathcal{H}=\frac{1}{2} \int\left(\Pi|\nabla \phi|^{2}-\left|\int^{\rho} \frac{\Pi}{\rho_{1}} d \rho_{1}\right|^{2}\right) d \mathbf{r} d \rho .
$$

The first term in this Hamiltonian clearly corresponds to the kinetic energy of the flow; that the second term is in fact the potential energy follows from the simple calculation

$$
\begin{gathered}
\frac{1}{2}\left|\int^{\rho} \frac{\Pi}{\rho_{1}} d \rho_{1}\right|^{2} d \rho=\frac{1}{2}\left|\int^{z} d z\right|^{2} d \rho= \\
=\frac{1}{2} z^{2} d \rho=-\rho z d z+d\left(\frac{1}{2} \rho z^{2}\right),
\end{gathered}
$$

so

$$
-\int_{\rho\left(z_{t}\right)}^{\rho\left(z_{b}\right)} \frac{1}{2}\left|\int^{\rho} \frac{\Pi}{\rho_{1}} d \rho_{1}\right|^{2} d \rho=\int_{z_{b}}^{z_{t}} \rho z d z-\left.\frac{1}{2} \rho z^{2}\right|_{z_{b}} ^{z_{t}},
$$

where $b$ and $t$ stand for bottom and top respectively, and the boundary conditions are usually such that the integrated term at the end is a constant. 


\subsection{Linear Shallow Waters in a Rotating Environment}

In a rotating environment, the linearized shallow-water equations are

$$
\begin{aligned}
\eta_{t}+\nabla \cdot \vec{u} & =0, \\
\vec{u}_{t}+\nabla \eta+\vec{u}^{\perp} & =0 .
\end{aligned}
$$

Here

$$
\vec{u}=\left(\begin{array}{c}
u \\
v
\end{array}\right)
$$

is the velocity field, and

$$
\vec{u}^{\perp}=\left(\begin{array}{r}
-v \\
u
\end{array}\right) .
$$

The Coriolis parameter $f$ has been absorbed in the nondimensionalization of time, so it is effectively equal to one.

These equations do not preserve vorticity, so irrotationality cannot be assumed. However, they preserve the potential vorticity

$$
q=v_{x}-u_{y}-\eta .
$$

The assumption corresponding to irrotationality in the non-rotating case is therefore that of zero potential vorticity, i.e. $q=0$. We can in fact generalize this hypothesis, and consider an arbitrary, though constant, potential vorticity. We shall employ such generalization when we consider internal waves in a rotating environment. In order to exploit this assumption, it is convenient to decompose the flow into a potential and a divergence-free part:

$$
\vec{u}=\nabla \phi+\nabla^{\perp} \psi,
$$

where

$$
\nabla^{\perp}=\left(\begin{array}{r}
-\partial_{y} \\
\partial_{x}
\end{array}\right)
$$

In terms of $\phi$ and $\psi$, the equations take the form

$$
\eta_{t}+\Delta \phi=0
$$




$$
\begin{aligned}
\phi_{t}+\eta-\psi & =0, \\
\psi_{t}+\phi & =0 .
\end{aligned}
$$

The condition of zero potential vorticity takes the form

$$
q=v_{x}-u_{y}-\eta=\Delta \psi-\eta=0,
$$

so the system above reduces to

$$
\begin{aligned}
\eta_{t}+\Delta \phi & =0 \\
\phi_{t}+\eta-\Delta^{-1} \eta & =0 .
\end{aligned}
$$

This system is Hamiltonian, with canonical variables $\phi$ and $\eta$, and Hamiltonian

$$
\mathcal{H}=\frac{1}{2} \int\left(\left|\nabla \phi+\nabla \Delta^{-1} \eta\right|^{2}+\eta^{2}\right) d x .
$$

Again, the Hamiltonian agrees with the total energy of the system.

\subsection{Rotating Nonlinear Shallow Waters}

The fully nonlinear equations for shallow waters in a rotating environment are

$$
\begin{aligned}
h_{t}+\nabla \cdot(h \vec{u}) & =0, \\
\vec{u}_{t}+(\vec{u} \cdot \nabla) \vec{u}+\nabla h+\vec{u}^{\perp} & =0 .
\end{aligned}
$$

The statement of conservation of potential vorticity now takes the form (Sec 12-2 in [28])

$$
\frac{D}{D t}\left(\frac{1+v_{x}-u_{y}}{h}\right)=0
$$

(That is: the total vorticity of a vertical column of water divided by its height remains constant as the column moves.) The unperturbed state has

$$
q=\frac{1+v_{x}-u_{y}}{h}=q_{0},
$$


where $q_{0}$ is an arbitrary (prescribed) potential vorticity, so this is the hypothesis to make for the analogue of irrotational flows:

$$
q_{0} h=1+v_{x}-u_{y} .
$$

We introduce the potentials $\phi$ and $\psi$ as in (16), and use the fact that

$$
(\vec{u} \cdot \nabla) \vec{u}=\frac{1}{2} \nabla\left|\nabla \phi+\nabla^{\perp} \psi\right|^{2}+\Delta \psi\left(\nabla^{\perp} \phi-\nabla \psi\right),
$$

to rewrite $(24)$ as

$$
\nabla \phi_{t}+\nabla^{\perp} \psi_{t}+\frac{1}{2} \nabla\left|\nabla \phi+\nabla^{\perp} \psi\right|^{2}+\Delta \psi\left(\nabla^{\perp} \phi-\nabla \psi\right)+\nabla h+\nabla^{\perp} \phi-\nabla \psi=0 .
$$

Taking the divergence and the two-dimensional $\operatorname{curl} \nabla^{\perp}$. of the above equations, we obtain the following pair:

$$
\begin{aligned}
\phi_{t}+\frac{1}{2}\left|\nabla \phi+\nabla^{\perp} \psi\right|^{2}+\Delta^{-1} \nabla \cdot\left[\Delta \psi\left(\nabla^{\perp} \phi-\nabla \psi\right)\right]+h-\psi & =0, \\
\psi_{t}+\Delta^{-1} \nabla^{\perp} \cdot\left[\Delta \psi\left(\nabla^{\perp} \phi-\nabla \psi\right)\right]+\phi & =0 .
\end{aligned}
$$

By noticing that

$$
\begin{gathered}
-\psi=\Delta^{-1} \nabla \cdot\left(\nabla^{\perp} \phi-\nabla \psi\right), \\
\phi=\Delta^{-1} \nabla^{\perp} \cdot\left(\nabla^{\perp} \phi-\nabla \psi\right),
\end{gathered}
$$

we can rewrite these equations, together with (23) in the form

$$
\begin{aligned}
h_{t}+\nabla \cdot\left(h\left(\nabla \phi+\nabla^{\perp} \psi\right)\right) & =0, \\
\phi_{t}+\frac{1}{2}\left|\nabla \phi+\nabla^{\perp} \psi\right|^{2}+\Delta^{-1} \nabla \cdot\left[(1+\Delta \psi)\left(\nabla^{\perp} \phi-\nabla \psi\right)\right]+h & =0, \\
\psi_{t}+\Delta^{-1} \nabla^{\perp} \cdot\left[(1+\Delta \psi)\left(\nabla^{\perp} \phi-\nabla \psi\right)\right] & =0 .
\end{aligned}
$$

The constraint (26) on the potential vorticity takes the form

$$
1+\Delta \psi=q_{0} h
$$


under which the equations above reduce to the pair

$$
\begin{aligned}
h_{t}+\nabla \cdot\left(h\left(\nabla \phi+\nabla^{\perp} \Delta^{-1}\left(q_{0} h-1\right)\right)\right) & =0 \\
\phi_{t}+\frac{1}{2}\left(\nabla \phi+\nabla^{\perp} \Delta^{-1}\left(q_{0} h-1\right)\right)^{2}+ & \\
q_{0} \Delta^{-1} \nabla \cdot\left[h\left(\nabla^{\perp} \phi-\nabla \Delta^{-1}\left(q_{0} h-1\right)\right)\right]+h & =0 .
\end{aligned}
$$

These equations are Hamiltonian, with conjugate variables $\phi$ and $h$, and Hamiltonian

$$
\mathcal{H}=\frac{1}{2} \int\left(h\left|\nabla \phi+\nabla^{\perp} \Delta^{-1}\left(q_{0} h-1\right)\right|^{2}+h^{2}\right) d \mathbf{r},
$$

representing again the sum of kinetic and potential energies.

\subsection{Nonlinear Internal Waves in a Rotating Environment}

The equations for long internal waves in a rotating environment are particularly simple when written in

the isopycnal coordinates $(x, y, \rho, t)$; they take the form in (11) with an extra term $\vec{u}^{\perp}$ due to the Coriolis force:

$$
\begin{aligned}
\frac{D \vec{u}}{D t}+\vec{u}^{\perp}+\nabla \frac{1}{\rho} \int^{\rho} \int^{\rho_{2}} \frac{\Pi-\Pi_{0}}{\rho_{1}} d \rho_{1} d \rho_{2} & =0, \\
\Pi_{t}+\nabla \cdot(\Pi \vec{u}) & =0 .
\end{aligned}
$$

where

$$
\Pi_{0}=\Pi_{0}(\rho)
$$

is a reference stratification profile, that we introduce here for future convenience.

The expression for the potential vorticity in these coordinates is

$$
q=\frac{1+v_{x}-u_{y}}{\Pi},
$$

and it satisfies

$$
\frac{D q}{D t}=0
$$


Notice that the advection of potential vorticity in (31) takes place exclusively along isopycnal surfaces. Therefore, an initial distribution of potential vorticity which is constant on isopycnals, though varying across them, will never change. Hence we shall propose that

$$
q=q_{0}(\rho)
$$

where $q_{0}(\rho)$ is an arbitrary function; i.e., one may assign any constant potential vorticity to each isopycnal surface. This is a highly nontrivial extension of the irrotational waves of the previous sections. Extending our description further to include general distributions of potential vorticity, varying even within surfaces of constant density, would necessarily complicate its Hamiltonian formulation, making it loose its natural simplicity. In fact, the problem of interaction between vorticity and waves is that of fully developed turbulence, which escapes the scope of our description. However, the "pancake-like" distributions of potential vorticity that we propose are common in stratified fluids, particularly the ocean and the atmosphere. They arise due to the sharp contrast between the magnitudes of the turbulent diffusion along and across isopycnals. Thus potential vorticity is much more rapidly homogenized along isopycnals than throughout the fluid, yielding the "pancakes". As we show below, even waves super-imposed on such a general and realistic distribution of potential vorticity admit a rather simple Hamiltonian description.

In order to isolate the wave dynamics satisfying the constraint (32), we decompose the flow into a potential and a divergence-free part as in (16). In terms of the potentials $\phi$ and $\psi,(32)$ and (31) yield

$$
1+\Delta \psi=q_{0} \Pi
$$

and, repeating the same steps as in nonlinear rotating shallow waters, the equations in (29) reduce to the pair

$$
\begin{aligned}
& \Pi_{t}+\nabla \cdot\left(\Pi\left(\nabla \phi+\nabla^{\perp} \Delta^{-1}\left(q_{0} \Pi-1\right)\right)\right)=0, \\
& \phi_{t}+\frac{1}{2}\left|\nabla \phi+\nabla^{\perp} \Delta^{-1}\left(q_{0} \Pi-1\right)\right|^{2} \\
&+\Delta^{-1} \nabla \cdot\left[q_{0} \Pi\left(\nabla^{\perp} \phi-\nabla \Delta^{-1}\left(q_{0} \Pi-1\right)\right)\right] \\
&+\frac{1}{\rho} \int^{\rho} \int^{\rho_{2}} \frac{\Pi-\Pi_{0}}{\rho_{1}} d \rho_{1} d \rho_{2}=0 .
\end{aligned}
$$


This pair is Hamiltonian, with conjugated variables $\phi$ and $\Pi$, i.e. it can be written as

$$
\Pi_{t}=\frac{\delta \mathcal{H}}{\delta \phi}, \quad \phi_{t}=-\frac{\delta \mathcal{H}}{\delta \Pi} .
$$

where the Hamiltonian is given by

$$
\mathcal{H}=\int\left[\frac{1}{2} \Pi\left|\nabla \phi+\nabla^{\perp} \Delta^{-1}\left(q_{0} \Pi-1\right)\right|^{2}-\frac{1}{2}\left|\int^{\rho} \frac{\Pi-\Pi_{0}}{\rho_{1}} d \rho_{1}\right|^{2}\right] d \rho d \vec{r} .
$$

Again, this Hamiltonian represents the sum of the kinetic and potential energy of the flow.

Notice the similarity of our description of internal waves with the Hamiltonian formulation for freesurface waves introduced in $[29,30]$. There, it was shown that the free-surface displacement and the three-dimensional velocity potential evaluated at the free surface are canonical conjugate variables. In our case, the canonical conjugate variables are also a displacement and a velocity potential, though the velocity potential in (36) is for the two-dimensional flow along isopycnal surfaces, and the displacement is the relative distance between neighboring isopycnal surfaces, as described above.

Looking back, we could have included some vorticity from early on; there was no need to take it equal to zero, as the last section shows. In shallow waters, it could have been any constant; in internal waves, any function of the density. It is clear though that, if one wanted to include arbitrary vorticity distributions, one would need to go fully Lagrangian, to exploit the fact that vorticity is preserved along particle paths. This would make the Hamiltonian structure less appealingly simple.

The key steps taken here for finding a simple Hamiltonian structure for internal waves, could be summarized as follows:

1. To consider long waves in hydrostatic balance. This, together with the choice of isopycnal coordinates, leads to a system of equations formally equivalent to an infinite collection of coupled shallow-water systems. This analogy allows us to generalize the relatively simple Hamiltonian structure of irrotational shallow-waters to the richer domain of internal waves. 
2. To decouple waves from vorticity, by assuming the latter to be either zero, constant or uniform along isopycnal surfaces, with an arbitrary dependence on depth. This is facilitated by the choice of a flow description in isopycnal coordinates.

3. To realize that the potential $\phi$ is a good candidate canonical variable, and that its conjugate is the height $h$ for shallow waters, and the surrogate $\Pi$ for density in the isopycnal formulation of internal waves.

4. To introduce nonlocal operators into the Hamiltonian. These arise naturally from the "elliptic" constraints of hydrostatic balance and layered potential vorticity. Despite its unusual look, the Hamiltonian is invariably just the sum of the standard kinetic and potential energies, integrated over the domain.

The assumptions of hydrostatic balance and horizontally uniform background vorticity and shear, which simplify notoriously the description of the flows, are quite realistic for a wide range of ocean waves.

\section{Weak turbulence theory}

In this section, we apply the formalism of wave turbulence theory to derive a kinetic equation, describing the time evolution of the energy spectrum of internal waves. In order to do this, we need to assume that the waves are weakly nonlinear perturbations of a background state. In principle, we could adopt for this state an arbitrary background distribution of (layered) potential vorticity, vertical shear and stratification. To make the derivation clear, however, we focus here on the case with zero shear and zero potential vorticity, and a stratification profile with constant buoyancy frequency. Even though the mechanics for deriving the kinetic equation in the more general setting are entirely similar (though more cumbersome), the tools at our disposal for finding relevant exact solutions to these equations, which we actually use in section 4, are only applicable to the case with the simplest background.

To leading order in the perturbation, we obtain linear waves, with amplitudes modulated by the nonlinear interactions. These linear waves have, in general, a complex vertical structure (they are eigen- 
functions of a differential eigenvalue problem), but reduce, in our case, to sines and cosines [31].

Let us now take (36) and rewrite it in dimensional form:

$$
\mathcal{H}=\int\left[\frac{1}{2} \Pi\left|\nabla \phi+\nabla^{\perp} \Delta^{-1}\left(q_{0} \Pi-f\right)\right|^{2}-\frac{g}{2}\left|\int^{\rho} \frac{\Pi-\Pi_{0}}{\rho_{1}} d \rho_{1}\right|^{2}\right] .
$$

Here $f$ is the Coriolis parameter, $g$ is the acceleration due to gravity. Note that

$$
[\Pi]=\text { Length }, \quad[\phi]=\frac{\text { Length }^{2}}{\text { Time }} .
$$

The potential vorticity is, in dimensional form,

$$
q=\frac{f+v_{x}-u_{y}}{\Pi} .
$$

In the calculations that follow, we shall consider flows which are perturbations of a state at rest, stratified but without vorticity. When this is the case, $v_{x}-u_{y}$ is zero to leading order, and we have the following relation between the potential vorticity profile $q_{0}$ and the stratification profile $\Pi_{0}$ :

$$
q_{0}(\rho)=\frac{f}{\Pi_{0}(\rho)} .
$$

Moreover, the definition of $\Pi$ implies that

$$
\Pi_{0}=-\frac{g}{N^{2}},
$$

where $N(\rho)$ is the buoyancy frequency, which we shall consider here to be a constant.

For the subsequent calculations it will be convenient to decompose the potential $\Pi$ into its equilibrium value and its deviation from it. Therefore let us redefine

$$
\Pi \rightarrow \Pi_{0}+\Pi \text {. }
$$

Then the Hamiltonian takes the following form:

$$
\mathcal{H}=\int d \vec{r} d \rho\left[\frac{1}{2}\left(-\frac{g}{N^{2}}+\Pi\right)\left|\nabla \phi-\frac{f N^{2}}{g} \nabla^{\perp} \Delta^{-1} \Pi\right|^{2}-\frac{g}{2}\left|\int^{\rho} \frac{\Pi}{\rho_{1}} d \rho_{1}\right|^{2}\right] .
$$


It can be represented as a sum of a quadratic and a cubic part:

$$
\begin{array}{r}
\mathcal{H}=\mathcal{H}_{\text {linear }}+\mathcal{H}_{\text {nonlinear }}, \\
\mathcal{H}_{\text {linear }}=\int d \vec{r} d \rho\left[-\frac{g}{2 N^{2}}\left|\nabla \phi-\frac{N^{2} f}{g} \nabla^{\perp} \Delta^{-1} \Pi\right|^{2}-\frac{g}{2}\left|\int^{\rho} \frac{\Pi}{\rho_{1}} d \rho_{1}\right|^{2}\right], \\
\mathcal{H}_{\text {nonlinear }}=\frac{1}{2} \int d \vec{r} d \rho \Pi\left|\nabla \phi-\frac{N^{2} f}{g} \nabla^{\perp} \Delta^{-1} \Pi\right|^{2}
\end{array}
$$

Let us use the Fourier transformation:

$$
\begin{aligned}
\Pi(\vec{r}, \rho) & =\frac{1}{(2 \pi)^{3 / 2}} \int \Pi_{\vec{p}} e^{i \vec{R} \vec{p}} d \vec{p}, \\
\phi(\vec{r}, \rho) & =\frac{1}{(2 \pi)^{3 / 2}} \int \phi_{\vec{p}} e^{i \vec{R} \vec{p}} d \vec{p}, \\
\vec{p} & =(\vec{k}, m), \quad \vec{R}=(\vec{r}, \rho) .
\end{aligned}
$$

Note that the operator $\nabla^{\perp} \Delta^{-1}$ has a simple representation in Fourier space:

$$
\nabla^{\perp} \Delta^{-1} \Pi(\vec{R})=-\frac{i}{(2 \pi)^{3 / 2}} \int d \vec{p} \frac{\vec{k}^{\perp}}{k^{2}} e^{i \vec{p} \vec{R}} \Pi_{\vec{p}}, \quad \vec{k}^{\perp}=\left(-k_{y}, k_{x}\right) .
$$

Since in the ocean, $\rho$ deviates from its equilibrium value $\rho_{0}$ by no more then $3 \%$, it is natural to make the Boussinesq approximation, replacing the density by a reference value $\rho_{0}$ :

$$
\frac{g}{2}\left|\int^{\rho} \frac{\Pi}{\rho_{1}} d \rho\right| \simeq \frac{g}{2 \rho_{0}}\left|\int^{\rho} \Pi d \rho\right| .
$$

Then

$$
\begin{array}{r}
\mathcal{H}_{\text {linear }}=-\frac{1}{2} \int d \vec{p}\left(\frac{g}{N^{2}} k^{2}\left|\phi_{\vec{p}}\right|^{2}+\left(\frac{N^{2} f^{2}}{g k^{2}}+\frac{g}{\rho_{0}^{2} m^{2}}\right)\left|\Pi_{\vec{p}}\right|^{2}\right), \\
\mathcal{H}_{\text {nonlinear }}=\frac{1}{2} \int d \vec{p}_{1} d \vec{p}_{2} d \vec{p}_{3} \delta\left(\vec{p}_{1}+\vec{p}_{2}+\vec{p}_{3}\right)\left(-\vec{k}_{2} \cdot \vec{k}_{3} \Pi_{\vec{p}_{1}} \phi_{\vec{p}_{2}} \phi_{\vec{p}_{3}}-\frac{N^{4} f^{2}}{g^{2}} \frac{\vec{k}_{2} \cdot \vec{k}_{3}}{k_{2}^{2} k_{3}^{2}} \Pi_{\vec{p}_{1}} \Pi_{\vec{p}_{2}} \Pi_{\vec{p}_{3}}-\right. \\
\left.2 \frac{N^{2} f}{g} \frac{\vec{k}_{2} \cdot \vec{k}_{3}^{\perp}}{k_{3}^{2}} \Pi_{\vec{p}_{1}} \Phi_{\vec{p}_{2}} \Pi_{\vec{p}_{3}}\right) .
\end{array}
$$

From now on it will be convenient to use the following short-hand notation: 
1. $\int d 123$ instead of $d \vec{p}_{1} d \vec{p}_{2} d \vec{p}_{3}$,

2. $\delta_{1+2+3}$ instead of $\delta\left(\vec{p}_{1}+\vec{p}_{2}+\vec{p}_{3}\right)$,

3. $\Pi_{i}$ and $\Phi_{i}$ instead of $\Pi_{\mathbf{p}_{\mathbf{i}}}$ and $\Phi_{\mathbf{p}_{\mathbf{i}}}$.

Then the last formula can be written in a more compact form:

$$
\begin{array}{r}
\mathcal{H}_{\text {nonlinear }}=\frac{1}{2} \int d 123 \delta_{1+2+3}\left(-\vec{k}_{2} \cdot \vec{k}_{3} \Pi_{1} \phi_{2} \phi_{3}-\right. \\
-\frac{N^{4} f^{2}}{g^{2}} \frac{\vec{k}_{2} \cdot \vec{k}_{3}}{k_{2}^{2} k_{3}^{2}} \Pi_{1} \Pi_{2} \Pi_{3}- \\
\left.2 \frac{N^{2} f}{g} \frac{\vec{k}_{2} \cdot \vec{k}_{3}^{\perp}}{k_{3}^{2}} \Pi_{1} \Phi_{2} \Pi_{3}\right)
\end{array}
$$

The canonical equations of motions (12) form a pair of real equations. Their Fourier transformation gives a pair of two complex equations, yet not independent. To reduce this pair to one complex equation, one performs the transformation

$$
\begin{gathered}
\phi_{\vec{p}}=\frac{i}{\sqrt{2} \sqrt{f_{\vec{p}}}}\left(a_{\vec{p}}-a_{-\vec{p}}^{*}\right), \\
\Pi_{\vec{p}}=\frac{\sqrt{f_{\vec{p}}}}{\sqrt{2}}\left(a_{\vec{p}}+a_{-\vec{p}}^{*}\right) .
\end{gathered}
$$

Here $f_{\vec{p}}$ is a real, positive and even, otherwise arbitrary function.

This transformation turns the pair of canonical equation of motion (12) into a single equation for the complex variable $a_{\vec{p}}$ :

$$
i \frac{\partial}{\partial t} a_{\mathbf{p}}=\frac{\partial \mathcal{H}}{\partial a_{\mathbf{p}}^{*}}
$$

The following choice of $f_{\vec{p}}$

$$
f_{\vec{p}}=\sqrt{\frac{g k^{2}}{N^{2}}\left(\frac{N^{2} f^{2}}{g k^{2}}+\frac{g}{\rho_{0}^{2} m^{2}}\right)^{-1}}
$$

diagonalizes the quadratic part of a Hamiltonian, bringing it to the following form:

$$
\mathcal{H}_{\text {linear }}=\int \omega_{\vec{p}}\left|a_{\vec{p}}\right|^{2} d \vec{p},
$$


where $\omega_{\vec{p}}$ is the dispersion relation for linear internal waves in isopycnal coordinates:

$$
\omega_{\vec{p}}=\sqrt{f^{2}+\frac{g^{2} k^{2}}{\rho_{0}^{2} m^{2} N^{2}}} .
$$

(In the more familiar Eulerian framework, the dispersion relation transforms into

$$
\omega_{\vec{p}}=\sqrt{f^{2}+\frac{N^{2} k^{2}}{m_{*}^{2}}},
$$

where $m_{*}$, the vertical wavenumber in $z$ coordinates, is given by $m_{*}=-\frac{g}{\rho_{0} N^{2}} m$.)

With such a choice of $f_{p}$ the transformations (44) take the following form:

$$
\begin{aligned}
\phi_{\vec{p}} & =\frac{i N \sqrt{\omega_{\vec{p}}}}{\sqrt{2 g} k}\left(a_{\vec{p}}-a_{-\vec{p}}^{*}\right), \\
\Pi_{\vec{p}} & =\frac{\sqrt{g} k}{\sqrt{2 \omega_{\vec{p}}} N}\left(a_{\vec{p}}+a_{-\vec{p}}^{*}\right) .
\end{aligned}
$$

In terms of $a_{\vec{k}}$, the Hamiltonian (42) reads

$$
\begin{array}{r}
\mathcal{H}=\int \omega_{p}\left|a_{\vec{p}}\right|^{2} d \vec{p}+ \\
\int V_{\vec{p}_{1} \vec{p}_{2} \vec{p}_{3}}\left(a_{\vec{p}_{1}}^{*} a_{\mathbf{p}_{2}}^{*} a_{\vec{p}_{3}}+a_{\vec{p}_{1}} a_{\vec{p}_{2}}^{*} a_{\vec{p}_{3}}^{*}\right) \delta_{\vec{p}_{1}-\vec{p}_{2}-\vec{p}_{3}} d \vec{p}_{123}+ \\
\int U_{\vec{p}_{1} \vec{p}_{2} \vec{p}_{3}}\left(a_{\vec{p}_{1}}^{*} a_{\mathbf{p}_{2}}^{*} a_{\vec{p}_{3}}^{*}+a_{\vec{p}_{1}} a_{\vec{p}_{2}} a_{\vec{p}_{3}}\right) \delta_{\vec{p}_{1}+\vec{p}_{2}+\vec{p}_{3}} d \vec{p}_{123} .
\end{array}
$$

This is a standard three-wave Hamiltonian of wave turbulence theory. The calculation of the interaction coefficients is a straightforward task, yielding

$$
\begin{aligned}
V_{23}^{1} & =I_{23}^{1}+J_{23}^{1}+K_{23}^{1}, \\
I_{23}^{1} & =-\frac{N}{4 \sqrt{2 g}}\left(\frac{\vec{k}_{2} \cdot \vec{k}_{3}}{k_{2} k_{3}} \sqrt{\frac{\omega_{2} \omega_{3}}{\omega_{1}}} k_{1}+\frac{\vec{k}_{1} \cdot \vec{k}_{3}}{k_{1} k_{3}} \sqrt{\frac{\omega_{1} \omega_{3}}{\omega_{2}}} k_{2}+\frac{\vec{k}_{1} \cdot \vec{k}_{2}}{k_{1} k_{2}} \sqrt{\frac{\omega_{1} \omega_{2}}{\omega_{3}}} k_{3}\right), \\
J_{23}^{1} & =\frac{N f^{2}}{4 \sqrt{2 g \omega_{1} \omega_{2} \omega_{3}}}\left(\frac{\vec{k}_{2} \cdot \vec{k}_{3}}{k_{2} k_{3}} k_{1}-\frac{\vec{k}_{1} \cdot \vec{k}_{3}}{k_{1} k_{3}} k_{2}-\frac{\vec{k}_{1} \cdot \vec{k}_{2}}{k_{1} k_{2}} k_{3}\right), \\
K_{23}^{1} & =\frac{i f N}{\sqrt{2 g}} \frac{\vec{k}_{2} \cdot \vec{k}_{3}^{\perp}}{k_{1} k_{2} k_{3}}\left(\sqrt{\frac{\omega_{2}}{\omega_{1} \omega_{3}}}\left(k_{1}^{2}-k_{3}^{2}\right)+\sqrt{\frac{\omega_{1}}{\omega_{2} \omega_{3}}}\left(k_{2}^{2}-k_{3}^{2}\right)+\sqrt{\frac{\omega_{3}}{\omega_{1} \omega_{2}}}\left(k_{2}^{2}-k_{1}^{2}\right)\right),
\end{aligned}
$$


where we have used the fact that $\overrightarrow{k_{1}}=\overrightarrow{k_{2}}+\overrightarrow{k_{3}}$.

Following wave turbulence theory, one proposes a perturbation expansion in the amplitude of the nonlinearity. This expansion gives to leading order, linear waves. Then one allows the amplitude of the waves to be slowly modulated by resonant nonlinear interactions. This modulation is described by an approximate kinetic equation [32] for the "number of waves" or wave-action $n_{\mathbf{p}}$, defined by

$$
n_{\mathbf{p}} \delta\left(\mathbf{p}-\mathbf{p}^{\prime}\right)=\left\langle a_{\mathbf{p}}^{*} a_{\mathbf{p}^{\prime}}\right\rangle
$$

This kinetic equation is the classical analog of the Boltzmann collision integral. The basic ideas for writing down the kinetic equation to describe how weakly interacting waves share their energies go back to Peierls. The modern theory has its origin in the works of Hasselmann [33,34], Benney and Saffmann [35], Kadomtsev [36], Zakharov [29,32,37], and Benney and Newell $[38,39]$. The derivation of kinetic equations using the wave turbulence formalism can be found, for instance, in $[32,40]$. For the three-wave Hamiltonian (48), the kinetic equation reads:

$$
\begin{gathered}
\frac{d n_{\mathbf{p}}}{d t}=\pi \int\left|V_{p p_{1} p_{2}}\right|^{2} f_{p 12} \delta_{\mathbf{p}-\mathbf{p}_{1}-\mathbf{p}_{\mathbf{2}}} \delta_{\omega_{\mathbf{p}}-\omega_{\mathbf{p}_{1}}-\omega_{\mathbf{p}_{\mathbf{2}}}} d \mathbf{p}_{12}, \\
\left.-2 \pi \int\left|V_{p_{1} p p_{2}}\right|^{2} f_{1 p 2} \delta_{\mathbf{p}_{1}-\mathbf{p}-\mathbf{p}_{\mathbf{2}}} \delta_{\omega_{\mathbf{p}_{1}}-\omega_{\mathbf{p}}-\omega_{\mathbf{p}_{2}}}\right) d \mathbf{p}_{12}
\end{gathered}
$$

where $f_{p 12}=n_{\mathbf{p}_{1}} n_{\mathbf{p}_{\mathbf{2}}}-n_{\mathbf{p}}\left(n_{\mathbf{p}_{1}}+n_{\mathbf{p}_{\mathbf{2}}}\right)$.

Assuming horizontal isotropy, one can average (50) over all horizontal angles, obtaining

$$
\begin{array}{r}
\frac{d n_{p}}{d t}=\frac{1}{k} \int\left(R_{12}^{k}-R_{k 2}^{1}-R_{1 k}^{2}\right) d k_{1} d k_{2} d m_{1} d m_{2}, \\
R_{12}^{k}=\Delta_{k 12}^{-1} \delta\left(\omega_{p}-\omega_{p_{1}}-\omega_{p_{2}}\right) f_{12}^{k}\left|V_{12}^{k}\right|^{2} \delta_{m-m_{1}-m_{2}} k k_{1} k_{2}, \\
\Delta_{k 12}^{-1}=\left\langle\delta\left(\mathbf{k}-\mathbf{k}_{\mathbf{1}}-\mathbf{k}_{\mathbf{2}}\right)\right\rangle \equiv \int \delta\left(\mathbf{k}-\mathbf{k}_{\mathbf{1}}-\mathbf{k}_{\mathbf{2}}\right) d \theta_{1} d \theta_{2}, \\
\Delta_{k 12}=\frac{1}{2} \sqrt{2\left(\left(k k_{1}\right)^{2}+\left(k k_{2}\right)^{2}+\left(k_{1} k_{2}\right)^{2}\right)-k^{4}-k_{1}^{4}-k_{2}^{4}} .
\end{array}
$$

\section{Kolmogorov Spectra in the High Frequency Limit}

Once the kinetic equation is derived, it is natural to search for its stationary solutions. Typically, kinetic equations admit two classes of exact stationary solutions: thermodynamic equilibrium and Kolmogorov 
flux solutions, with the latter corresponding to a direct cascade of energy -or other conserved quantitiestoward the higher modes. The fact that the thermodynamic equilibrium -or equipartition of energy$n_{\mathbf{p}}=1 / \omega_{\mathbf{p}}$ is a stationary solution of (51) can be seen by inspection. On the other hand, the solutions, corresponding to pure Kolmogorov spectra are much more subtle and only emerge after one has exploited scaling symmetries of the dispersion relation and the coupling coefficient via what is now called the Zakharov transformation [32].

In this section, we carry out such procedure in the high-frequency limit, where the effects of the Earth's rotation are negligible. The spectrum that we find, expanding on previous work described in [20], is not far from the classical empirical formula of Garrett and Munk.

Since the system under consideration has cylindrical, rather then spherical symmetry, stationary solutions of the kinetic equation should also have cylindrical symmetry. Things are further complicated by the fact that our dispersion law (46) is not scale invariant, and neither is the interaction matrix element (49). However, in the high frequency limit $\omega \gg f,(46)$ becomes

$$
\omega_{\mathbf{p}} \equiv \omega_{\mathbf{k}, m} \simeq \frac{g}{N \rho_{0}} \frac{k}{|m|},
$$

Furthermore, in this limit, the matrix element (49) retains only it first term, $I_{23}^{1}$. This is due to the fact that the second $J_{23}^{1}$ and third $K_{23}^{1}$ terms are proportional to $f^{2}$ and $f$ respectively, and $f$ is negligible in the high frequency limit.

Indeed if one changes variables in (49) so that

$$
\omega_{i}=N \xi_{i}
$$

rescaling the frequencies in terms of the buoyancy frequency $N$, and similarly one introduces

$$
\vec{k}_{i}=\vec{\kappa}_{i} / L
$$

i.e. nondimensionalizing the horizontal wavevectors in terms of some distance $L$ to be determined, then

$$
V_{23}^{1}=I_{23}^{1}+J_{23}^{1}+K_{23}^{1}
$$




$$
\begin{aligned}
I_{23}^{1} & =-\frac{N^{\frac{3}{2}}}{4 \sqrt{2 g} L}\left(\frac{\vec{\kappa}_{2} \cdot \vec{\kappa}_{3}}{\kappa_{2} \kappa_{3}} \sqrt{\frac{\xi_{2} \xi_{3}}{\xi_{1}}} \kappa_{1}+\frac{\vec{\kappa}_{1} \cdot \vec{\kappa}_{3}}{\kappa_{1} \kappa_{3}} \sqrt{\frac{\xi_{1} \xi_{3}}{\xi_{2}}} \kappa_{2}+\frac{\vec{\kappa}_{1} \cdot \vec{\kappa}_{2}}{\kappa_{1} \kappa_{2}} \sqrt{\frac{\xi_{1} \xi_{2}}{\xi_{3}}} \kappa_{3}\right) \\
J_{23}^{1} & =\frac{N^{3 / 2}}{4 L \sqrt{2 g}} \frac{f^{2}}{N^{2}} \frac{1}{\sqrt{2 g \xi_{1} \xi_{2} \xi_{3}}}\left(\frac{\vec{\kappa}_{2} \cdot \vec{\kappa}_{3}}{\kappa_{2} \kappa_{3}} \kappa_{1}-\frac{\vec{\kappa}_{1} \cdot \vec{\kappa}_{3}}{\kappa_{1} \kappa_{3}} \kappa_{2}-\frac{\vec{\kappa}_{1} \cdot \vec{\kappa}_{2}}{\kappa_{1} \kappa_{2}} \kappa_{3}\right) \\
K_{23}^{1} & =i \frac{N^{3 / 2}}{L \sqrt{2 g}} \frac{f}{N} \frac{\vec{\kappa}_{2} \cdot \vec{\kappa}_{3}^{\perp}}{\kappa_{1} \kappa_{2} \kappa_{3}}\left(\sqrt{\frac{\xi_{2}}{\xi_{1} \xi_{3}}}\left(\kappa_{1}^{2}-\kappa_{3}^{2}\right)+\sqrt{\frac{\xi_{1}}{\xi_{2} \xi_{3}}}\left(\kappa_{2}^{2}-\kappa_{3}^{2}\right)+\sqrt{\frac{\xi_{3}}{\xi_{1} \xi_{2}}}\left(\kappa_{2}^{2}-\kappa_{1}^{2}\right)\right)
\end{aligned}
$$

Note that $K_{23}^{1}$ is proportional to $(f / N)$ and $J_{23}^{1}$ is proportional to $(f / N)^{2}$. Taking into account that, in the real ocean, $f / N \simeq 1 / 100$ we see that the $K_{23}^{1}$ and $J_{23}^{1}$ terms could safely be neglected from the matrix element in the high frequency limit.

We reproduce here for completeness the derivation of the Kolmogorov solution found in [20]. Let us assume that $n_{\mathbf{p}}$ is given by the power-law anisotropic distribution

$$
n_{\mathbf{k}, m}=k^{x}|m|^{y} .
$$

To find the values of the exponents $x$ and $y$ we will require that (52) is a stationary solution to (51) in the high frequency limit. We shall use a version of Zakharov's transformation [29,37] introduced for cylindrically symmetrical systems by Kuznetsov in [41]. Namely, let us subject the integration variables in the second term $R_{k 2}^{1}$ in (51) to the following transformation:

$$
k_{1}=k^{2} / k_{1}^{\prime}, m_{1}=m^{2} / m_{1}^{\prime}, k_{2}=k k_{2}^{\prime} / k_{1}^{\prime}, m_{2}=m m_{2}^{\prime} / m_{1}^{\prime} .
$$

Then $R_{k 2}^{1}$ becomes $R_{12}^{k}$ multiplied by a factor

$$
\left(\frac{k_{1}}{k}\right)^{-6-2 x}\left(\frac{m}{m_{1}}\right)^{2+2 y}
$$

Furthermore, let us subject the third term $R_{1 k}^{2}$ in (51) to the similar conformal transformation:

$$
k_{1}=k k_{1}^{\prime} / k_{2}^{\prime}, m_{1}=m m_{1}^{\prime} / m_{2}^{\prime}, k_{2}=k^{2} / k_{2}^{\prime}, m_{2}=m^{2} / m_{2}^{\prime} .
$$

Then $R_{1 k}^{2}$ becomes $R_{12}^{k}$ multiplied by a factor

$$
\left(\frac{k_{2}}{k}\right)^{-6-2 x}\left(\frac{m}{m_{2}}\right)^{2+2 y} .
$$


Therefore (51) can be written as

$$
\begin{aligned}
\frac{d n_{\mathbf{p}}}{d t}= & \frac{1}{k} \int R_{12}^{k}\left(1-\left(\frac{k_{1}}{k}\right)^{-6-2 x}\left(\frac{m}{m_{1}}\right)^{2+2 y}-\right. \\
& \left.\left(\frac{k_{2}}{k}\right)^{-6-2 x}\left(\frac{m}{m_{2}}\right)^{2+2 y}\right) d k_{1} d k_{2} d m_{1} d m_{2} .
\end{aligned}
$$

We see that the particular choice $-6-2 x=2+2 y=1$, which gives $x=-7 / 2, \quad y=-1 / 2$, makes the right-hand side of (51) vanish due to the delta function in the frequencies, corresponding to energy conservation. The resulting wave action and spectral energy distributions are given by

$$
\begin{array}{r}
n_{\mathbf{k}, m}=|\mathbf{k}|^{-7 / 2}|m|^{-1 / 2}, \\
E_{\mathbf{k}, m}=k \omega_{\mathbf{k}, m} n_{\mathbf{k}, m}=|\mathbf{k}|^{-3 / 2}|m|^{-3 / 2},
\end{array}
$$

This solution corresponds to the flux of energy from the large to the small scales. Direct calculations show that this solution is local, i.e. collision integral converges on this solution.

We shall next compare the spectrum just derived with the classic formula by Garrett and Munk [1-3], which synthesizes in compact form many measurements of oceanic internal wave turbulence.

To this end, let us first write the total energy of the system as the integral of a spectral energy density:

$$
E=\int E(\mathbf{k}, m) d k d m
$$

Note that $E(\mathbf{k}, m)$ is integrated over scalars $k$ and $m$. Garrett and Munk proposed the following empirical expression for $E(k, m)$ :

$$
E(k, m)=\frac{3 f N E m / m^{*}}{\pi\left(1+\frac{m}{m^{*}}\right)^{5 / 2}\left(N^{2} k^{2}+f^{2} m^{2}\right)} .
$$

Here $E$ is a constant, quantifying the total energy content of the internal wave spectrum, $k=|\mathbf{k}|$, and $m^{*}$ is a reference vertical wavenumber determined from observations. Using the dispersion relation for internal waves, the GM spectrum can be transformed from wave-number space $(k, m)$ into frequencyhorizontal wavenumber space $(k, \omega)$. The integral of $E(k, \omega)$ over $k$ yields the moored spectrum

$$
E(\omega)=2 f E\left(\pi\left(1-(f / \omega)^{2}\right)^{1 / 2} \omega^{2}\right)^{-1}
$$


with an $1 / \omega^{2}$ dependence away from the inertial frequency that appears prominently in moored observations.

For $|m|>>m^{*}$ and $|\omega|>>f$, the Garrett-Munk spectrum (56) becomes

$$
E(k, m) \simeq\left(k^{2} m^{3 / 2}\right)^{-1}
$$

By contrast, the spectrum that we obtained above using the Wave Turbulence formalism is

$$
E(k, m) \simeq\left(k^{3 / 2} m^{3 / 2}\right)^{-1}
$$

The small difference between the two spectra may be due to a variety of reasons. Two possibilities that we find highly plausible are the neglected effects of the Earth's rotation, and those of breaking waves. An exploration of these possibilities, however, lies outside the scope of the present article.

\section{Conclusions}

We have developed a quite general, natural Hamiltonian formalism for internal waves in a stratified, rotating environment. Our formulation gains much in simplicity, by restricting consideration to flows in hydrostatic balance, superimposed on a vertically arbitrary, but horizontally uniform shear and vorticity fields. The resulting Hamiltonian inherits much of the structure of the shallow-water equations, though with one extra vertical dimension. The use of isopycnal coordinates, whereby the depth $z$ is replaced by the density $\rho$ as the independent vertical coordinate, allows for a straightforward separation of the dynamics of waves and vorticity, by assuming the latter to be uniform on surfaces of constant density.

This Hamiltonian formulation allows us to derive a kinetic equation for the time evolution of the spectral energy density. In the limit of high frequencies, when the effects of the rotation of the Earth loose significance, an exact steady solution to this kinetic equation can be found, corresponding to the direct cascade of energy toward the short scales. This Kolmogorov-like spectrum is surprisingly close to the empirically based prediction of Garrett and Munk.

Further challenges suggested by the work reported here, include extending the Kolmogorov solutions found in the high-frequency limit, to cover the full range of frequencies of internal waves, including those 
comparable to the inertial frequency $f$. Also, the effects of breaking waves on the energy spectrum needs to be addressed; it could potentially help explain the difference between the exponent of the spectrum found here and that of Garrett and Munk.

\section{References}

[1] Garrett C.J.R and Munk W.H. Space time scales of internal waves. Geophysical Fluid Dynamics, $3: 225-264,1972$.

[2] Garrett C.J.R and Munk W.H. Space time scales of internal waves, progress report. J. Geophys. Res, 80:281-297, 1975.

[3] Garrett C.J.R and Munk W.H. Internal waves in the ocean. Annual Review of Fluid mechanics, 11:339-369, 1979.

[4] J.R. Ledwell, E.T. Montgomery, K.L. Polzin, L.C. Laurent, R.W. Schmitt, and J.M. Toole. Evidence for enhanced mixing over rough topography in the abyssal ocean. Nature, 403:179-82, 2000.

[5] J.M. Toole, R.W. Schmitt, K.L. Polzin, and E.Kunze. Near-boundary mixing above the flanks of a midlatitude seamount. Journal of Geophysical Research, 102:947-59, 1997.

[6] K.L. Polzin, N.S. Oakey, J.M. Toole, and R.W.Schmitt. Fine structure and microstructure characteristics across the northwest atlantic subtropical front. Journal of Geophysical Research, 101:14111-21, 1996.

[7] K. Polzin. Statistics of the richardson number: mixing models and finestructure. Journal of Physical Oceanography, 26:1409-25, 1996.

[8] R.A. Weller, D.L. Rudnick, C.C. Eriksen, K.L.Polzin, N.S. Oakey, J.W.Toole, R.W. Schmitt, and R.T.Pollard. Forced ocean response during the frontal air-sea interaction experiment. Journal of Geophysical Research, 95:8611-38, 1991. 
[9] Dirk Olbers. Nonlinear energy transfer and the energy balance of the intenal waves fiel in the deep ocean. Journal of Fluid mechanics, 74:375-399, 1976.

[10] Peter Muller, Greg Holloway, Frank Henyey, and Neil Pomphrey. Nonlinear interaction among internal gravity waves. Review of Geophysics, 24:493-536, 1986.

[11] Pelinovskii E.N. and Raevsky M.A. Weak turbulence of internal ocean waves. Izvestiya Akademii Nauk SSSR, Fizika Atmosfery i Okeana, translated in Izvestiya Academy of Sciences USSR, Atmospheric and Oceanic Physics, 13:187-93, 1977.

[12] A.G.Voronovich. Hamiltonian formalism for internal waves in the ocean. Izvestiya, Atmospheric and Oceanic Physics, 16:52-57, 1979.

[13] Iu. Z. Miropolskii. Dynamics of Internal Gravity Waves in the Ocean (Atmospheric and Oceanographic Sciences Library, V. 24). Kluwer Academic Publishers, 2001, translation from Russian of "Dinamika vnutrennih voln v okeane", Gidrometeoizdat 1981.

[14] P. Caillol and Z. Zeitlin. Kinetic equations and stationary energy spectra of weakly nonlinear internal gravity waves. Dynamics of Atmosphere and Ocean, 32:81-112, 2000.

[15] C.O.Hines. Geophys Astrophys Fluid Dynamics, 96:1-30, 2002.

[16] C.O. Hines and O. Colin. Theory of the eulerian tail in the spectra of atmospheric and oceanic internal gravity waves. Journal of Fluid Mechanics, 448:289-313, 2001.

[17] I. Chunchuzov. On the high wavenumber form of the eulerian internal wave spectrum in the atmosphere. Journal of Atmospheric Sciences, 59:1753-1774, 2002.

[18] D.Broutman. On doppler-spreading models of internal waves. Geophys Research Letters, 24:2813$2816,1997$.

[19] S.D. Eckermann. sentropic advection by gravity waves: quasi-universal m/sup -3/ vertical wavenumber spectra near the onset of instability. Geophys Res Letters, 26:201-204, 1999. 
[20] Yuri V. Lvov and Esteban Tabak. Hamiltonian formalism and the garrett-munk spectrum of internal waves in the ocean. Physical Review Letters, 87:168501, 2001.

[21] Michael Milder. Hamiltonian description of internal waves. Journal of FLuid mechanics, 119:269$282,1982$.

[22] F.S. Henyey. Hamiltonian description of stratified fluid dynamics. Physics of Fluids, 26:40-7, 1983.

[23] F.S. Henyey and C.R. Graham. Clebsch representation near points where the vorticity vanishes. Physics of Fluids, 12:744-6, 2000.

[24] V Zeitlin. Vorticity and waves: geometry of a phase-space and the problem of normal variables. Physics Letters A, 164:177-183, 1992.

[25] I. Gjaja and D.D. Holm. Self-consistent hamiltonian dynamics of wave mean-flow interaction for a rotating stratified incompressible fluid. Physica D, 98:343-78, 1996.

[26] P. J. Morrison. Hamiltonian description of the ideal fluid. Rev. Mod. Phys., 70:467-521, 1998.

[27] R. Salmon. Hamilotonian fluid mechanics. Journal Annual review of fluid mechanics, 20:225-256, 1988.

[28] Benoit Cushmar-Roisin. Introduction to Geophysical Fluid dynamics. Prentice Hall, 1994.

[29] V.E.Zakharov. Stability of periodic waves of finite amplitude on a surface of deep fluid. J.Appl.Mech. Tech. Phys, 2:190-198, 1967.

[30] J.W. Miles. Hamiltonian formulations for surface waves. Applied Scientific Research, 37:103-10, 1981.

[31] A.E.Gill. Atmosphere-Ocean Dynamics. Academic Press, 1982.

[32] V.E. Zakharov, V.S. L'vov, and G.Falkovich. Kolmogorov Spectra of Turbulence. Springer-Verlag, 1992. 
[33] K.Hasselmann. On the nonlinear energy transfer in a gravity wave spectrum. part $i$. general theory. J. Fluid Mech., 12:481, 1962.

[34] K.Hasselmann. On the nonlinear energy transfer in a gravity wave spectrum. part $i$. conservation theorems, wave-particle analogy, irreversibility. J. Fluid Mech., 15, 1962.

[35] D.J. Benney and P.Saffmann. Nonlinear interaction of random waves in a dispersive medium. Proc Royal. Soc, 289:301-320, 1966.

[36] B.B. Kadomtsev. Plasma Turbulence. Academic Press, New York, 1965.

[37] V. E. Zakharov. The instability of waves in nonlinear dispersive media. Sov. Phys. JETP, 24(4):740$744,1967$.

[38] J. Benney and A.C. Newell. Random wave closure. Studies in Appl. Math., 48:1, 1969.

[39] A. C. Newell. Solitons in mathematics and physics. SIAM, Philadelphia, 1985.

[40] A. J. Majda, D. W. McLaughlin, and E. G. Tabak. A one-dimensional model for dispersive wave turbulence. J. Nonlin. Sci., 6:9-44, 1997.

[41] E.A.Kuznetsov. Zh. Eksp.Teor.Fiz, 62, 1972. 